\title{
Effect of salt reduced bread alone or with dietary counselling on 24-hour excretion of sodium, potassium and sodium/potassium ratio
}

\author{
Nanna Louise Riis ${ }^{1,2}$, Kirsten Bjørnsbo ${ }^{2}$, Anne Dahl Lassen ${ }^{1}$, Ellen Trolle ${ }^{1}$, Amalie Frederiksen ${ }^{2}$, \\ Anne Helms Andreasen ${ }^{2}$ and Ulla Toft ${ }^{2}$ \\ ${ }^{1}$ Division of Risk Assessment and Nutrition, National Food Institute, Technical University of Denmark, Lyngby, \\ Denmark and \\ ${ }^{2}$ Center for Clinical Research and Prevention, Frederiksberg Hospital, Frederiksberg, Denmark
}

\begin{abstract}
Excess dietary sodium plays an important role in hypertension, a leading risk factor for cardiovascular disease (CVD). To counteract some of the negative effects of sodium, an increased intake of potassium has shown beneficial effects on blood pressure. In fact, the sodium to potassium ratio might be a stronger risk factor for CVD than either sodium or potassium alone. The objective of the present study was to estimate the effect of A) a salt reduction strategy (providing salt reduced bread) and B) a salt reduction and potassium promoting strategy (providing salt reduced bread combined with dietary counselling), on intake of sodium, potassium and the sodium/potassium ratio. The study is a 4-month cluster randomized controlled trial with families randomly assigned to either intervention A, intervention $\mathrm{B}$ or control. Participants in intervention A received bread gradually reduced in salt content from $1.2 \mathrm{~g}$ salt/ $100 \mathrm{~g}$ to $0.6 \mathrm{~g}$ salt $/ 100 \mathrm{~g}$ in ryebread and $0.4 \mathrm{~g}$ salt $/ 100 \mathrm{~g}$ in wheat bread. Participants in intervention B received the same salt reduced bread and in addition dietary counselling on how to eat food with less salt and more potassium. The control group received standard bread $(1.2 \mathrm{~g}$ salt $/ 100 \mathrm{~g})$. Sodium and potassium intake were measured through three consecutive 24-hour urine measurements in adults and one in children at baseline and follow-up. Changes in outcomes were assessed using linear mixed models. Eighty-nine families, including 155 adults (46.5\% men) and 156 children (51.9\% boys), from the suburb of Copenhagen, Denmark, participated in the study. The sodium/potassium ratio was significantly reduced by $-0.57 \pm 0.19 \mathrm{mmol} / \mathrm{L}(\mathrm{P}=0.003)$ in intervention $\mathrm{B}$ compared to control group, resulting from a non-significant lower sodium and increased potassium intake. No significant differences were found between intervention A and control group (preliminary results). These findings support future actions to reduce salt intake, focusing on a combination of structural and educational strategies.
\end{abstract}

\section{Conflict of Interest}

There is no conflict of interest 